\title{
Complex signatures of selection for the melanogenic loci TYR, TYRPI and DCT in humans
}

Santos Alonso*1, Neskuts Izagirre ${ }^{1}$, Isabel Smith-Zubiaga ${ }^{2}$, Jesús Gardeazabal ${ }^{3}$, José Luís Díaz-Ramón³ ${ }^{3}$ José Luís Díaz-Pérez ${ }^{3}$, Diana Zelenika ${ }^{4}$, María Dolores Boyano ${ }^{5}$, Nico Smit ${ }^{6}$ and Concepción de la Rúa ${ }^{1}$

\author{
Address: ${ }^{1}$ Dept. Genetics, Physical Anthropology and Animal Physiology. University of the Basque Country. Barrio Sarriena s/n. 48940 Leioa, \\ Bizkaia, Spain, ${ }^{2}$ Dept. Zoology and Animal Cell Biology. University of the Basque Country. Barrio Sarriena s/n. 48940 Leioa, Bizkaia, Spain, \\ ${ }^{3}$ Dermatology Service. Cruces Hospital. Plaza de Cruces s/n, 48903, Cruces-Barakaldo, Bizkaia, Spain, ${ }^{4}$ Centre National de Génotypage (CNG). 2 \\ Rue Gaston Cremieux. CP 5721. 91057 Evry Cedex, France, ${ }^{5}$ Dept. Cell Biology and Histology. University of the Basque Country. Barrio Sarriena

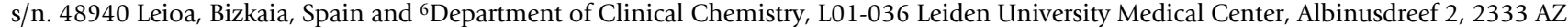 \\ Leiden, The Netherlands \\ Email: Santos Alonso* - santos.alonso@ehu.es; Neskuts Izagirre - neskuts.izagirre@ehu.es; Isabel Smith-Zubiaga - isabel.smith@ehu.es; \\ Jesús Gardeazabal - jesus.gardeazabal@ehu.es; José Luís Díaz-Ramón - secretaria.derma@hcru.osakidetza.net; José Luís Díaz- \\ Pérez - jldiaz@hcru.osakidetza.net; Diana Zelenika - zelenika@cng.fr; María Dolores Boyano - lola.boyano@ehu.es; \\ Nico Smit - N.P.M.Smit@lumc.nl; Concepción de la Rúa - conchi.delarua@ehu.es \\ * Corresponding author
}

Published: 29 February 2008

BMC Evolutionary Biology 2008, 8:74 doi:10.1 I86/I47|-2/48-8-74

This article is available from: http://www.biomedcentral.com/I47|-2/48/8/74

(C) 2008 Alonso et al; licensee BioMed Central Ltd.

This is an Open Access article distributed under the terms of the Creative Commons Attribution License (http://creativecommons.org/licenses/by/2.0), which permits unrestricted use, distribution, and reproduction in any medium, provided the original work is properly cited.

\begin{abstract}
Background: The observed correlation between ultraviolet light incidence and skin color, together with the geographical apportionment of skin reflectance among human populations, suggests an adaptive value for the pigmentation of the human skin. We have used Affymetrix UI 33a v2.0 gene expression microarrays to investigate the expression profiles of a total of 9 melanocyte cell lines ( 5 from lightly pigmented donors and 4 from darkly pigmented donors) plus their respective unirradiated controls. In order to reveal signatures of selection in loci with a bearing on skin pigmentation in humans, we have resequenced between 4 to $5 \mathrm{~kb}$ of the proximal regulatory regions of three of the most differently expressed genes, in the expectation that variation at regulatory regions might account for intraespecific morphological diversity, as suggested elsewhere.
\end{abstract}

Results: Contrary to our expectations, expression profiles did not cluster the cells into unirradiated versus irradiated melanocytes, or into lightly pigmented versus darkly pigmented melanocytes. Instead, expression profiles correlated with the presence of Bovine Pituitary Extract (known to contain $\alpha-M S H$ ) in the media. This allowed us to differentiate between melanocytes that are synthesizing melanin and those that are not. TYR, TYRPI and DCT were among the five most differently expressed genes between these two groups. Population genetic analyses of sequence haplotypes of the proximal regulatory flanking-regions included Tajima's D, HEW and DHEW neutrality tests analysis. These were complemented with EHH tests (among others) in which the significance was obtained by a novel approach using extensive simulations under the coalescent model with recombination. We observe strong evidence for positive selection for TYRPI alleles in 
Africans and for DCT and TYRPI in Asians. However, the overall picture reflects a complex pattern of selection, which might include overdominance for DCT in Europeans.

Conclusion: Diversity patterns clearly evidence adaptive selection in pigmentation genes in Africans and Asians. In Europeans, the evidence is more complex, and both directional and balancing selection may be involved in light skin. As a result, different non-African populations may have acquired light skin by alternative ways, and so light skin, and perhaps dark skin too, may be the result of convergent evolution.

\section{Background}

The pigmentation of the human skin is the result of a complex process by which the pigmentary biopolymer, melanin, is produced and packed in the melanosome (a specialized organelle of melanocytes) and is distributed to the surrounding epidermal keratynocytes. Based mainly on the study of mouse mutants, a description has been made of over 100 loci that are involved in the pigmentary phenotype [1].

Although a lot of work has been done on the biochemistry and cytology of pigmentation, the evolutionary genetic history of this phenotypic trait has been less investigated. Initially, the observed correlation between ultraviolet radiation (UVR) and skin color [2], together with the geographical apportionment of skin reflectance among human populations in the major continental regions [3], suggest that the evolution of human skin pigmentation has been adaptive. Early evolutionary studies focused on $M C 1 R$ diversity [4,5]. Although both these works observed a depletion of variability in Africans in comparison to Europeans and Asians, which was explained by purifying selection in Africans, the interpretation for the enhanced Euro-Asiatic diversity was different. Thus, while [4] claimed that diversifying selection has been responsible for the high diversity found in Eurasians, [5] invoked functional relaxation, and therefore neutral evolution, for this locus outside Africa. Phylogenetic comparison of MC1R primate sequences by [6] indicated that $M C 1 R$ has been subject to purifying selection.

Other genes have been associated with the pigmentary phenotype, such as the P locus (OCA2) [7], MATP (alias AIM1, SLC45A2) [8] or ASIP [9,10]. More recently, it has been suggested that different genes may be responsible for the light pigmentary phenotypes observed in different non-African populations. Accordingly, a derived allele in a SNP within SLC24A5 correlates with lighter skin in Europeans, but not with light skin in East Asians [11]. Additional support for this point came from the work by [12], who used public SNP databases to single out DCT as a pigmentation gene candidate for recent positive selection in the Chinese only. In addition, [13] found that while ASIP and OCA2 might play a shared role in shaping light and dark pigmentation across the globe, genes like
SLC24A5, MATP and TYR may have had a predominant role in the evolution of light skin in Europeans but not in East Asians. According to the authors, this provides compelling evidence that light skin has evolved independently in European and East Asian populations. Other genomewide scans for genes under recent positive selection from ascertained SNPs claimed several pigmentation genes (OCA2, MYO5A, DTNBP1 and TYRP) as candidates for adaptive selection in Europeans only [14], whereas [15] suggested that protection against the damaging effects of UVR might hold an adaptive value in Africans.

However, most of the genetics of pigmentation is based on the mouse as a model organism. In contrast to humans, melanocytes in adult murine skin are generally confined mainly to hair follicles (also to the external ear and tail) [16]. It is therefore likely that some of the pigmentation genetics learnt from mice may not be directly applicable to humans. In this context, we have used Affymetrix U133A 2.0 microarray analysis to investigate the gene expression profiles of different human melanocyte cell lines. This is expected to provide us with a list of loci involved in human skin pigmentation, which can be used as candidate loci for evolutionary inference. Taking these results into account, we have resequenced between 4 to $5 \mathrm{~kb}$ of the proximal regulatory regions of three genes of interest, namely, TYR, TYRP1 and DCT. These genes form a homogeneous unit, as they belong to the same family and coordinate the production of melanin from tyrosine. Thus, the rate-limiting enzyme in melanogenesis is tyrosinase (EC 1.14.18.1) (the product of TYR), which catalyses the conversion of tyrosine into dopaquinone [17]. Tyrosinase activity is required for the synthesis of two types of melanins, pheomelanins (red to yellow melanins) or the more photoprotective eumelanins (brown to black melanins). Pheomelanogenesis seems to be the default pathway in the absence of MC1R signaling, with low tyrosinase activity and a high concentration of cysteine. Instead, eumelanin synthesis requires $\alpha-\mathrm{MSH}$ binding to MC1R $[18,19]$, which transcriptionally activates tyrosinase and upregulates tyrosinase-related protein-1 (EC 1.14.18, the product of TYRP1) and DOPAchrome tautomerase (EC 5.3.2.3; the product of the DCT locus, formerly TYRP2 or TRP-2). Other genes like $A S P$, the $\alpha-M S H$ antagonist, slightly reduce tyrosinase 
activity and produce an almost complete loss of TYRP1 and DCT expression, thereby decreasing eumelanin synthesis [20].

We have focused on the proximal regulatory region, as it has been proposed that variation at regulatory regions may account for intraespecific morphological diversity [21-25]. We therefore resequenced 116 human chromosomes from diverse geographical origin, including Africans, Europeans (plus European melanoma patients), Asians and Australian Aborigines, in search of diversity patterns that could help us reconstruct the evolutionary history of human skin pigmentation.

\section{Results and discussion Microarray experiments}

Affymetrix U133a v2.0 microarrays were used to analyze five melanocyte cell lines from lightly pigmented donors and four from darkly pigmented donors, plus their respective unirradiated controls. Two additional cell lines from darkly pigmented donors did not yield RNA of sufficient quality for reverse transcription.

The first eigenvector for the SAM Pattern Discovery (unsupervised) analysis identified 5,404 probes as differentially expressed at an FDR of 4.65\% (see Additional file 1). Unexpectedly, expression values did not cluster the cells into unirradiated versus irradiated melanocytes, or into lightly pigmented versus darkly pigmented melanocytes. Instead, we observed a differentiation between the M1 and $\mathrm{M} 4$ cells on the one hand (M1: lightly pigmented donor, irradiated and unirradiated; M4: darkly pigmented donor, irradiated and unirradiated), and the rest of the cell lines on the other. One possible explanation points to the different growth media used. However, a precise comparison of the media is difficult because providers do not always disclose the detailed formulation of the commercial media. With the information available, we can speculate on the absence of melanocyte mitogens BPE and/or PMA in the M1 and M4 cell cultures as a possible explanation. Both PMA and BPE could be boosting proliferation and melanogenesis in the "rest of the cells" group. In fact, both M1 and M4 proved to be very slow growing. In this regard, BPE usually contains $\alpha-\mathrm{MSH}$, which is a strong stimulator of melanogenesis. It has been shown that the induced release of $\alpha$-MSH by UV light-irradiated keratinocytes (and to a much lesser extent, by irradiated melanocytes also) stimulates melanogenesis [26]. Along these lines, [27] reported also that human melanocytes cultured in media containing BPE do not respond to increasing concentrations of $\alpha-\mathrm{MSH}$, whereas the removal of BPE resulted in a significant reduction in melanocyte proliferation and melanogenesis, but restored responsiveness to melanotropins. However, for microarray experiments, the difficulty in growing melanocyte cultures without BPE may hamper the obtaining of the high cell numbers required. To confirm the effect of the presence/absence of BPE on the expression profiles of TYR, TYRP1 and DCT, we quantified by qPCR (see Material and Methods, Quantitative PCR section) the expression levels of these genes in one cell line that was grown in (a) Cascade growth medium 254 supplemented with 1\% HGMS (includes $\mathrm{BPE}$ ) vs. the same cell line grown in (b) Cascade medium plus PromoCell Supplement (no BPE). In this case all three genes suffered a reduction in their expression levels when grown under the second conditions (normalized ratios (conditions b) $/($ conditions a) TYR: $0.44 \pm 0.09$, TYRP1: $0.27 \pm 0.05$ and DCT: $0.05 \pm 0.01$ ) (3 replicates). We also investigated the effect of adding a known amount of BPE to the medium and thus we compared the expression levels of these when cell were grown in (c) PromoCell growth medium plus PromoCell Supplement vs. (d) PromoCell growth medium plus PromoCell Supplement and $0.2 \%(\mathrm{v} / \mathrm{v})$ of a $13 \mathrm{mg} / \mathrm{ml}$ solution of BPE. Addition of BPE led to an increase of the expression level of all three genes (normalized ratios (conditions $\mathrm{d}$ )/(conditions $\mathrm{c}$ ) TYR: $1.5 \pm 0.22$, TYRP1: $1.96 \pm 0.26$ and DCT: $1.93 \pm 0.74)$ ( 2 replicates). These results indicate that in melanocytes, BPE has a biological effect on the expression of, at least, TYR, TYRP1 and DCT.

In any case, the homogeneity found in the gene expression profiles of human melanocytes grown in BSE-containing media suggests that melanocytes from both light and dark pigmentation donors may have the same genetic ability to produce melanin if subject to the same level of external (paracrine) signaling molecules, at least above a certain concentration.

Interestingly enough, however, Gene Ontology analysis of differentially expressed genes between these two groups indicates that the term "melanin biosynthesis from tyrosine" is a significant, non-redundant, "biological process" term (FDR p-value 0.0197). Genes belonging to this biological term are overexpressed in the "rest of the cells" group. Among the top over-expressed probes we can find those corresponding to MLAN-A, TYR, TYRP1, DCT, GPR143, S100B, OCA2, SILV or SOX10, and further down the list, MITF, PAX3, KIT or SLC45A2 (MATP), genes known for their involvement in melanocyte function and development. MC1R is further down the list, but still shows an average $\log _{2}$ difference in gene expression of over 3 (overexpressed in the "rest of the cells" group). Rather unexpectedly, SLC24A5, a gene recently claimed as a major pigmentation gene in humans [11], does not appear in this list. Nevertheless, this list provides a good collection of candidate loci for further diversity analysis in human populations from which to obtain evolutionary inferences. Non-redundant GO terms (Biological processes) over-presented in the M1 and M4 cells group 
include: cell-adhesion (FDR p-value 1.3e-6), cell-cell signaling (FDR p-value 2.05e-5), inflammatory response (FDR p-value 2.05e-5), skeletal development (FDR pvalue 2.55e-4), chemotaxis (FDR p-value 6.7e-4), morphogenesis (FDR p-value 1.6e-3), sensory perception (FDR p-value 3.9e-3), organelle organization and biogenesis (FDR p-value 6.5e-3), RNA processing (FDR p-value $1.4 \mathrm{e}-2$ ), phosphate transport (FDR p-value 1.4e-2), cell surface receptor linked signal transduction (FDR p-value 1.9e-2), humoral defense mechanism (sensu Vertebrata) (FDR p-value 2.06e-2), reproductive organismal physiological process (FDR p-value 3.1e-2), DNA repair (FDR pvalue 3.1e-2), cellular macromolecule metabolism (FDR p-value 3.2e-2), metal ion transport (FDR p-value 3.3e-2) and protein metabolism (FDR p-value 3.8e-2).

Based on this information, we decided to resequence the proximal regulatory regions of the melanogenic loci TYR, TYRP1 and DCT in search of evidence for selection on these genes. It is expected that resequencing, rather than typing previously ascertained SNPs, will provide unbiased information that will help us to more faithfully reconstruct the evolutionary history of these pigmentation loci.

\section{Population diversity and neutrality tests from sequence data}

In the approximately $4 \mathrm{~kb}$ of the TYR upstream genomic region resequenced we have detected 20 SNPs in the global sample of 116 chromosomes. We have also detected two polymorphisms derived from runs of Ts at chr11:88548969 and chr11:88550172, one GA microsatellite between chr11:88549822.88550079, one polymorphic Alu insertion at chr11:88548585, which seems to belong to the Ya5 family, and three polymorphic indels, one of them involving $4 \mathrm{bp}$ and two involving $2 \mathrm{bp}$. None of these non-SNP polymorphisms was considered for the diversity analysis, partly because of their different mutational nature to SNPs and partly because of the intrinsic difficulty in sequencing through some of these regions. Out of the above 20 SNPs, one falls within a putative CAAT box located about 200 bp upstream the translation initiation codon. None of them falls within other known cis-regulatory regions [23]. These polymorphisms assemble into 29 different haplotypes, whose genealogical relationships are shown in Figure 1.

In the approximately $5 \mathrm{~kb}$ of the TYRP1 upstream genomic region resequenced we have detected 29 SNPs. Polymorphisms derived from a run of Ts at chr9:12679614 and from a run of As at chr9:12680664 as well as a polymorphic rearrangement involving a $18 \mathrm{bp}$ insertion found at chr9:12683765, were not considered in the analysis for the same reasons as above. None of the 29 SNPs fell within known cis-regulatory regions [23]. These polymorphisms assemble into 32 different haplotypes,
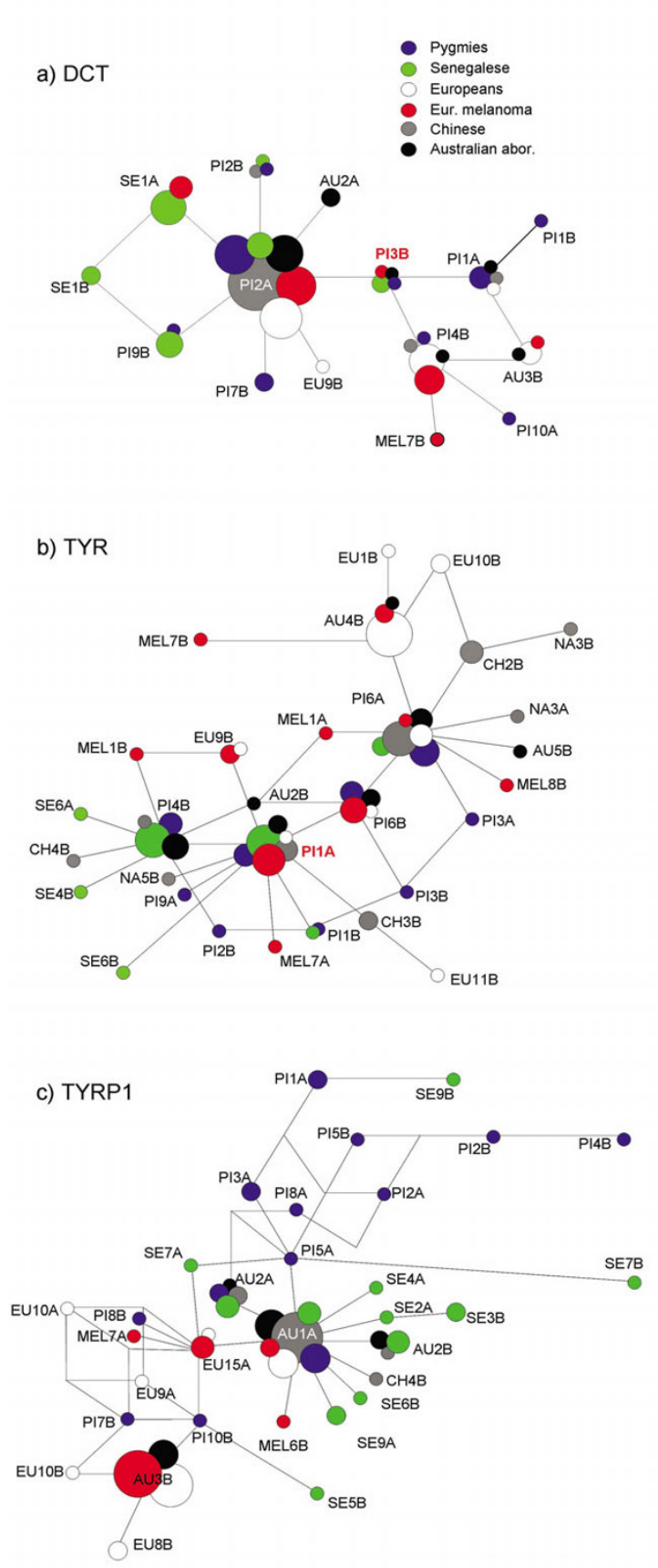

Figure I

Median-Joining network describing the genealogical relationships among the haplotypes found in the resequenced 5 '-flanking regions of TYR, TYRPI and DCT. Areas are proportional to absolute frequency. Blue circles: Pygmies; white circles: Europeans; red circles: European melanoma patients; gray circles: Chinese; green circles: Senegalese; and black circles: Australian Aborigines. The ancestral allele for DCT and TYR is indicated in red. The ancestral allele for TYRPI could not be unequivocally identified, but most likely falls two mutational steps away from AUIA. 
whose genealogical relationships are shown in Figure 1. Haplotype frequencies and genotypes are available as supplementary material (see Additional file 2)

For the $4.1 \mathrm{~kb}$ region upstream from $D C T$, we have detected a total of 9 SNPs plus one indel (involving $1 \mathrm{bp}$ ), which was not considered for the sequence analysis for the same reasons as above. None of the SNPs falls within the known cis-regulatory regions located in the approximately 500 bp upstream of the transcription initiation point $[23,28]$. These polymorphisms assemble into 15 different haplotypes, whose genealogical relationships are shown in Figure 1.

Detected SNPs and indels have been submitted to dbSNP and will be publicly available in Build 130 (see Additional file 3).
After removing polymorphic positions, we estimated the divergence between a chimp sequence and the human sequence. The estimated divergence for DCT was 0.01 (95\% C.I. $0.0074-0.0136) ; 0.0104 \quad(95 \% \quad$ C.I. 0.0075-0.0134) for TYRP1 and 0.0152 (95\% C.I. 0.0114-0.0198) for TYR. These values fall within normal average neutral values [29] and suggest that no substantial evolutionary acceleration has occurred in these genomic regions since the human-chimp split.

Diversity measures for all samples are summarized in Table 1. Overall, both DCT and TYRP1 show higher haplotype diversity in the African populations analyzed, whereas TYR shows similar levels of haplotype diversity across populations.

Table I: Diversity parameters and neutrality test for the resequenced regions of TYR, TYRPI and DCT

\begin{tabular}{|c|c|c|c|c|c|c|}
\hline & Pygmies & Senegal & Australian Ab. & Chinese & Europeans & Melanoma \\
\hline \multicolumn{7}{|c|}{ Number of segregating sites, S: } \\
\hline$D C T$ & 6 & 4 & 4 & 4 & 4 & 4 \\
\hline TYR & 5 & 8 & 5 & 7 & 6 & 7 \\
\hline TYRPI & $\mathrm{II}$ & 21 & 5 & 3 & 6 & 4 \\
\hline \multicolumn{7}{|c|}{ Number of Haplotypes, h: } \\
\hline DCT & 9 & 6 & 4 & 4 & 5 & 6 \\
\hline$T Y R$ & 9 & 7 & 7 & 7 & 8 & 10 \\
\hline TYRPI & 13 & 12 & 4 & 4 & 7 & 5 \\
\hline \multicolumn{7}{|c|}{ Haplotype diversity, Hd (SD): } \\
\hline$D C T$ & $0.79(0.09)$ & $0.82(0.05)$ & $0.66(0.12)$ & $0.28(0.13)$ & $0.70(0.07)$ & $0.74(0.07)$ \\
\hline TYR & $0.89(0.04)$ & $0.77(0.06)$ & $0.88(0.06)$ & $0.85(0.06)$ & $0.70(0.1)$ & $0.88(0.05)$ \\
\hline TYRPI & $0.93(0.04)$ & $0.94(0.03)$ & $0.7 \mid(0.08)$ & $0.36(0.13)$ & $0.71(0.08)$ & $0.57(0.12)$ \\
\hline \multicolumn{7}{|c|}{ Nucleotide diversity, $\mathrm{Pi}(\mathrm{SD})\left(* 10^{-4}\right)$} \\
\hline DCT & $3.8(0.7)$ & $3.1(0.3)$ & $3(0.7)$ & $1.2(0.6)$ & $3.5(0.4)$ & $3.5(0.5)$ \\
\hline TYR & $5(5)$ & $3.8(0.8)$ & $4.9(0.6)$ & $5.5(0.7)$ & $3.8(0.1)$ & $5.4(0.8)$ \\
\hline TYRPI & $7(1)$ & $6(1)$ & $4(1)$ & I (0) & $4(1)$ & $2(1)$ \\
\hline \multicolumn{7}{|c|}{ Theta (per site) from S, Theta-W (SDa) $\left(* 10^{-4}\right)$} \\
\hline DCT & $4.1(1.7)$ & $2.7(1.4)$ & $3.1(1.5)$ & $2.8(1.4)$ & $2.7(1.3)$ & $2.7(1.4)$ \\
\hline$T Y R$ & $3.8(1.7)$ & $6.1(2.2)$ & $4.3(1.9)$ & $5.3(2)$ & $4.5(1.8)$ & $5.3(2)$ \\
\hline TYRPI & $6(2)$ & $12(3)$ & $3(1)$ & $2(I)$ & $3(1)$ & $2(1)$ \\
\hline \multicolumn{7}{|c|}{ Average number of nucleotide differences, k: } \\
\hline DCT & 1.574 & 1.253 & 1.231 & 0.489 & 1.446 & 1.421 \\
\hline$T Y R$ & 1.832 & 1.4 & 1.802 & 2.032 & 1.403 & 1.989 \\
\hline TYRPI & 3.268 & 2.963 & 1.890 & 0.389 & $\mathrm{I} .827$ & 1.289 \\
\hline \multicolumn{7}{|c|}{ Tajima's D value } \\
\hline$D C T$ & -0.221 & 0.321 & -0.071 & -1.638 & 0.892 & 0.754 \\
\hline TYR & 0.917 & -1.278 & 0.506 & 0.098 & -0.458 & 0.027 \\
\hline TYRPI & 0.192 & -1.906 & 0.700 & -1.447 & 0.341 & 0.416 \\
\hline \multicolumn{7}{|c|}{ Tajima's D, p (D <= D obs) } \\
\hline$D C T$ & $0.39^{b}(0.37)^{c}$ & $0.67^{b}(0.59)^{c}$ & $0.52^{b}(-)^{c}$ & $0.04^{b}(0.03)^{c}$ & $0.84^{b}(0.65)^{c}$ & $0.8^{b}(0.62)^{c}$ \\
\hline$T Y R$ & $0.86^{b}(0.79)^{c}$ & $0.04^{b}(0.02)^{c}$ & $0.73^{b}(-)^{c}$ & $0.57^{b}(0.39)^{c}$ & $0.33^{b}(0.2 I)^{c}$ & $0.54^{b}(0.38)^{c}$ \\
\hline TYRPI & $0.64^{b}(0.49)^{c}$ & $\mid e-3^{b, d}(\mid e-3)^{c, d}$ & $0.8^{b}(-)^{c}$ & $0.09^{b}(0.04)^{c}$ & $0.67^{b}(0.44)^{c}$ & $0.69^{b}(0.5)^{c}$ \\
\hline \multicolumn{7}{|c|}{ DHEW (HEW) p-values } \\
\hline$D C T$ & $0.19(0.26)$ & $0.68(0.73)$ & $0.20(0.22)$ & $4 e-3^{e}\left(5 e-3^{f}\right)$ & $0.63(0.32)$ & $0.57(0.20)$ \\
\hline$T Y R$ & $0.75(0.8 \mathrm{I})$ & $0.33(0.43)$ & $0.62(0.69)$ & $0.27(0.12)$ & $0.1\left(3 e-3^{f}\right)$ & $0.52(0.63)$ \\
\hline TYRPI & $0.87(0.91)$ & $0.61(0.72)$ & $0.76(0.82)$ & $0.28(0.33)$ & $0.4(0.03)$ & $0.44(0.03)$ \\
\hline
\end{tabular}

a assuming free recombination.

b using the standard neutral model in DnaSP.

c using a demography-corrected neutral model.

d significant after multiple test correction, at an FDR of $5 \%$.

e significant after multiple test correction, at an FDR of $8 \%$

f significant after multiple test correction, at an FDR of $6 \%$. 
Among the non-African populations, the Chinese sample shows a substantial drop in diversity for both DCT and TYRP1. This drop in diversity is accompanied by significantly negative Tajima's D values for both DCT and TYRP1, although these values become non-significant after multiple-test correction (data not shown). DHEW and HEW tests are also significant for DCT in the Chinese (even after multiple-test correction, at an FDR of $8 \%$ and $6 \%$, respectively) (Table 1 ). These results suggest possible positive selection acting on alleles of these loci in the Chinese, in particular for DCT.

The European samples, however, show positive (although non-significant) Tajima's D values for DCT and TYRP1. Excluding population structure, which is unlikely to be substantial in our European sample, positive Tajima's D values can be obtained when some kind of diversityincreasing selection is operating [30]. Analysis of the HapMap data confirms the existence of SNPs (like rs9301959, rs7990565 or rs4773797) with higher than expected heterozygosity $(\mathrm{p}<0.01)$ in the extended DCT region in Caucasians. This observation is compatible with selection by overdominance. Europeans and European melanoma patients show some evidence for selection at TYRP1 (HEW test, see Table 1), but this is not significant after multipletest correction. Caucasians share their major TYRP1 haplotypes, AU1A and AU3B, with Australian Aborigines. The AU1A allele seems to pre-date the out-of-Africa expansion, since all African and non-African populations analyzed also share this haplotype. This suggests that this haplotype has little to do with pigmentation differences across populations. As regards AU3B, the recent claim that aboriginal Australians descend from the same African emigrant group as all other Eurasians [31] suggests an age for this shared allele of at least 50,000-70,000 years. Thus, despite the relatively high frequency of AU3B in Europeans, given the pigmentary differences between Australian Aborigines and Europeans and the lack of strong evidence for selection in them, it seems unlikely that this haplotype is associated with a light skin phenotype, at least in a simple way. In Europeans, only TYR shows some evidence for selection but only for the HEW test (significant after multiple-test correction at an FDR of $6 \%$ ). This is most likely due to the fact that HEW is more powerful than DHEW in detecting ongoing or recently completed selective sweeps [32]. The contrasting patterns between Tajima's D and HEW in TYR might indicate a partial sweep at this locus in the European (healthy) sample. This signature is not observed however in the sample of European melanoma patients. Interestingly, European melanoma patients show a frequency for AU4B that is markedly lower than that for healthy Europeans (Fischer's exact test p-value: 0.0025). However, this observation should be considered only as exploratory, as it is not our purpose in this work to conduct a formal test of association to melanoma.

For the African populations analyzed (Pygmies and Senegalese, both dark-skinned populations), the networks observed in Figure 1 suggest that the main factor responsible for the lack of strong negative Tajima's D values in the Pygmy sample is population structure. In fact, our Pygmy sample is composed of both Biaka and Mbuti Pygmies, who have been described as "substantially different genetically" [33]. This observation can explain the positive Tajima's D values found for the Pygmy sample [34].

In contrast, the Senegalese record a highly significant negative Tajima's D value for TYRP1 (significant even after multiple-test correction, at an FDR value of 5\%), which is suggestive of selection. The observation that neither DHEW nor HEW are significant for the African populations (Table 1) is however consistent, as DHEW and HEW are expected to have much less power than Tajima's D to detect selection after fixation of the advantageous allele when $\rho$ is no greater than $\theta$ [32], which is the case for the Senegalese TYRP1 diversity. It may be argued that the simulation parameters inferred from the Yoruba are not applicable to other African populations. In an attempt to offset the effects of a possible population expansion on Tajima's D p-value observed for the Senegalese, and in the absence of a precise demographic model for this population, we have run further simulations assuming for the Senegalese the demographic model used previously for the Europeans. This is expected to provide a reasonably conservative correction. Under this demographic scenario, the p-value for Tajima's D in the Senegalese is approximately 0.027 . Thus, we are inclined to believe that the negative Tajima's $\mathrm{D}$ in the case of the Senegalese TYRP1 diversity reflects the signature of an old selective process.

Lastly, the lack of signal in Australian Aborigines can be explained by the effect of drift: if the population that left Africa and colonized Australia was relatively small and relatively constant, whereby the effect of drift overcame the effect of selective pressure, then this signal of selection may have been lost.

\section{Other Selection tests based on the HapMap project data}

In order to detect selection in more recent evolutionary time windows [35], we have used the SNP frequency information available in the HapMap dataset to perform, firstly, a scan for SNPs with significant pairwise $\mathrm{F}_{\mathrm{ST}}$ values across these loci, and secondly, a search for longer than expected haplotypes within populations by means of the EHH test [36] (see also Material and Methods). 
$\mathrm{F}_{\mathrm{ST}}$ comparison is useful for detecting selection in a time window of less than 50,000 to 75,000 years [35]. After multiple-test correction, several SNPs were found to be positive across DCT and TYRP1 (including the flanking regions) for the HapMap Chinese+Japanese vs. HapMap Caucasians pairwise comparison (Table 2). Overall, our results agree with those by [12], although in our case, multiple-test correction makes non-significant additional pairwise comparisons that [12] found significant for their uncorrected $\mathrm{F}_{\mathrm{ST}}$ tests.

EHH tests can be useful to detect signatures of partial selective sweeps in a time window of less than 30,000 years [35]. We observed haplotypes with longer than expected $\mathrm{EHH}$ in the HapMap Chinese+Japanese sample for DCT and TYRP1, and in the HapMap Yoruba for TYRP1 (Table 3, Figure 2).

Using a different long-range haplotype statistic for DCT, [12] suggested the existence of a core SNP at rs2031526 whose bidirectional breakdown of haplotype homozygosity of the derived allele "A" evidenced selection. Our EHH approach (see Material and Methods), however, did not identify this SNP as a strong candidate core, perhaps because our procedure to declare significance (see Material and Method) is more stringent than that used by [12]. Instead, our DCT core haplotype "TCA", which is located around $11 \mathrm{~kb}$ upstream from rs2031526, overlapping an intronic conserved region in mammals, actually identifies a subset of the rs2031526 "A" haplogroup.

The core SNPs for TYRP1 HapMap Yoruba fall within a conserved region $(77 \%$ identity with the mouse orthologous sequence) that contains at least one potential transcription factor binding site for SOX10 (a known melanogenesis enhancer) and is placed less than $5 \mathrm{~kb}$ upstream of a putative TYRP1 enhancer sequence [37].

The relatively low frequencies of DCT core haplotype "TCA" in the Chinese+Japanese and of TYRP1 core haplotype "TGA" in the Yoruba (Table 3) suggest that we are detecting the signature of selection in young haplogroups only. $\mathrm{EHH}$ is expected to fail to detect the signature of selection in older haplotypes because of the decay of LD with time. However, at least in the case of TYRP1, the fact that both Tajima's D and EHH detect selection may indicate that the environmental pressure was probably old, and either continued to act for some time after the origin of this "TGA" allele and then ceased or is still ongoing.

Finally, the core SNPs for TYRP1 in the HapMap Chinese+Japanese fall astride a LTR (MER52A, family ERV1) and a LINE (L1MA2) element, around $15 \mathrm{~kb}$ upstream from the aforementioned core SNPs for TYRP1 in the HapMap Yoruba. Complete homozygosity for a core haplogroup "AA" extends well into the 5'-flanking region of the gene, and seems to constitute a major subset of allele

Table 2: HapMap genotyped SNPs showing significant $\mathbf{F}_{\mathbf{S T}}{ }^{\mathbf{a}}$

\begin{tabular}{|c|c|c|c|c|}
\hline Locus & SNP ID & Positionb & Gene region & $F_{S T}$ CEU vs ASIc \\
\hline \multirow[t]{15}{*}{ TYRPI } & rs/3293905 & Chr9:I2675943 & 5' flanking region & 0.679 \\
\hline & rs10756393 & Chr9:12682252 & 5' flanking region & 0.558 \\
\hline & rs2762462 & Chr9:12689776 & intron & 0.638 \\
\hline & rs2762463 & Chr9:1269|897 & intron & 0.671 \\
\hline & rs2733832 & Chr9:12694725 & intron & 0.581 \\
\hline & rs 2733833 & Chr9:12695095 & intron & 0.661 \\
\hline & rs2075509 & Chr9:12695219 & intron & 0.553 \\
\hline & rs2209277 & Chr9:12696236 & intron & 0.671 \\
\hline & rs683 & Chr9:12699305 & 3' UTR & 0.644 \\
\hline & rs2762464 & Chr9:12699586 & 3' UTR & 0.652 \\
\hline & rs910 & Chr9:12700035 & 3' UTR & 0.671 \\
\hline & rs 1063380 & Chr9:12700090 & 3' UTR & 0.671 \\
\hline & rs2733835 & Chr9:12702I57 & 3' flanking region & 0.581 \\
\hline & rs 12379024 & Chr9:12707405 & 3' flanking region & 0.599 \\
\hline & rs10491744 & Chr9:12710106 & $3^{\prime}$ flanking region & 0.599 \\
\hline \multirow[t]{5}{*}{$D C T$} & rsl3256II & Chrl3:93892386 & intron & 0.607 \\
\hline & rs|407995 & Chrl 3:938940I4 & intron & 0.594 \\
\hline & rs203I526 & Chrl 3:93898842 & intron & 0.607 \\
\hline & rs3782972 & Chrl 3:93901047 & intron & 0.641 \\
\hline & rs6492706 & Chrl 3:93937385 & $5^{\prime}$ flanking region & 0.468 \\
\hline
\end{tabular}

a Multiple-test corrected significant $\mathrm{F}_{\mathrm{ST}}$ values at an FDR of $5 \%$ (see Material and Methods). A total of 97 SNPs were analyzed.

b Coordinates from HapMap Data Rel. 2 Ia/Phasell, Jan07 on NCBI B35 assembly, dbSNP bI 25.

c only CEU vs. ASI show multiple-test corrected significant $F_{S T}$ values. CEU: HapMap North European Caucasians; ASI: HapMap Chinese plus Japanese. 

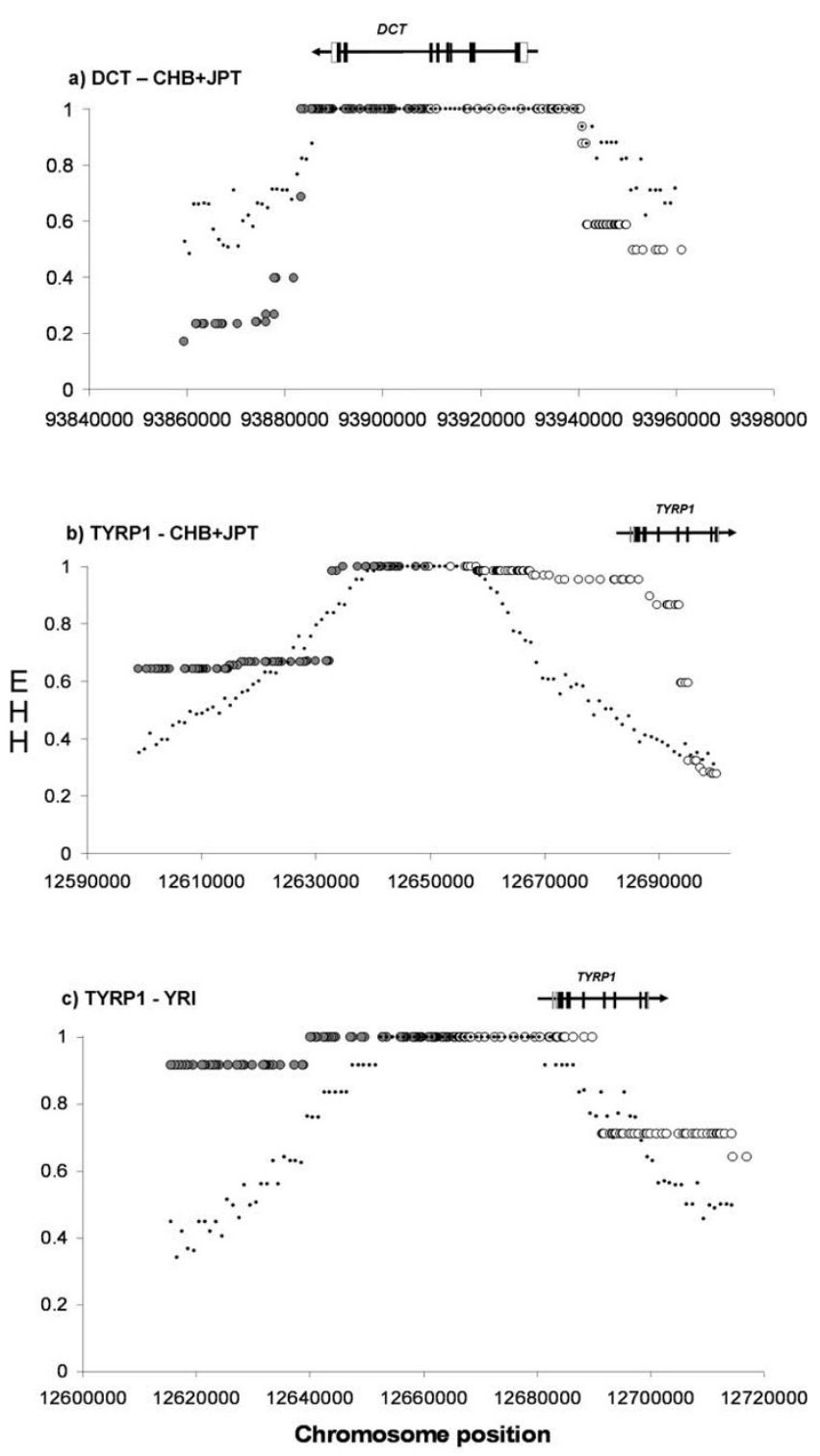

Figure 2

Extended Haplotype Homozygosity (EHH) tests. After scanning the HapMap data with Sweep (see Methods) candidate core haplotypes were tested for the significance of their extended haplotype homozygosity $(\mathrm{EHH})$. Circles represent the observed $\mathrm{EHH}$ values extending about $50 \mathrm{~kb}$ from the core haplotype in both directions, white for $3^{\prime}$ and gray for 5 '. Dots represent the corresponding $95 \%$ upper percentiles of the simulated EHH distributions. The approximate location of the lociwith respect to the core haplotypes is indicated on top: coding exons in black, UTRs in white, and the intervening lines represent the introns. The arrows indicate the direction of transcription. a) EHH for DCT in the HapMap Chinese+Japanese population; b) EHH for TYRPI in the HapMap Chinese+Japanese population and c) EHH for TYRPI in the HapMap Yoruba population.
AU1A. Its high frequency (see table 3) and long EHH correlates with the (weak) signal of selection detected by Tajima's D in the Chinese TYRP1 sequence diversity.

The proximity of the two core haplotypes for TYRP1 observed in two different populations, in combination with the long $\mathrm{EHH}$ that extends into the flanking region of the locus, and the evidence for selection on these two populations from the Tajima's D test, point to a region distally upstream of TYRP1 as the target region for selection on TYRP1 diversity.

\section{Conclusion}

It is well-established that dark skin is photoprotective (see [38] and references therein), but whether this photoprotective role represents an evolutionary advantage is less clear. Alternative mechanisms to explain a possible adaptive advantage of dark pigmentation involve concealment [39] or the protection of folate from UVR-induced photolysis [40]. However, in the light of the apparent vagaries that occur in the pigmentation of mammalian skin, including primates, others [41] suggest that dark skin may have outlived its usefulness. Selective mechanisms for light skin involve the facilitation of vitamin D3 synthesis [40].

The ability to detect selection from diversity patterns depends on many aspects, such as the demography of the population under study, the mutation and recombination rates of the locus, the dominance and selection coefficients of the advantageous allele, whether the advantageous allele has reached fixation (and how long ago) or not, and whether the favorable allele arose from a single mutation at the time of the selective pressure or was favored from standing variation [42]. Thus, we have used a battery of different tests to enhance the ability to detect selection, and we have been able to show the signature of directional selection particularly on TYRP1 in Africans, and on DCT and TYRP1 in Asians. We should bear in mind that the constitutive pigmentation of the skin is not exclusively dependent on melanin production. Other factors, such as the distribution of melanins within melanosomes, the melanosome secretion rate by melanocytes, the rate of melanosome ingestion by keratynocytes and the distribution of melanosomes within keratynocytes, are also major determinants of skin pigmentation ([43] and references therein). The question of how these mechanisms can be functionally linked to the observed diversity patterns in the melanogenic loci studiedcannot be answered with the present data. However, the observed patterns of diversity do seem to support the idea that selection has had a role in the evolutionary history of melanogenesis. 
Table 3: Haplotypes with longer than expected homozygosity (EHH test) for DCT and TYRPI.

\begin{tabular}{|c|c|c|c|c|c|c|c|}
\hline \multirow[t]{2}{*}{ Locus } & \multirow{2}{*}{$\begin{array}{l}\text { Core } \\
\text { SNPs IDs }\end{array}$} & \multirow[t]{2}{*}{ Chr position ${ }^{\mathrm{a}}$} & \multirow[t]{2}{*}{ Core haplotype } & \multicolumn{3}{|c|}{ frequencyc } & \multirow[t]{2}{*}{ pop } \\
\hline & & & & CEU & ASI & YRI & \\
\hline \multirow[t]{3}{*}{$D C T$} & rs9516418 & Chr|3:93909510 & TCA & $5 \%$ & $18 \%$ & $0 \%$ & ASI \\
\hline & rs9584234 & Chr|3:93909660 & $(101)^{b}$ & & & & \\
\hline & rs I 2877248 & Chrl 3:9390983 & & & & & \\
\hline \multirow[t]{2}{*}{ TYRPI } & rs 10809814 & Chr9:I2649098 & AA & $5 \%$ & $71 \%$ & $19 \%$ & ASI \\
\hline & rs 4741242 & Chr9:|264969| & $(10)^{b}$ & & & & \\
\hline \multirow[t]{3}{*}{ TYRPI } & rs1096075I & Chr9:12665264 & TGA & $0 \%$ & $0 \%$ & $20 \%$ & YRI \\
\hline & rs 10960752 & Chr9:12665284 & $(00 \mathrm{I})^{\mathrm{b}}$ & & & & \\
\hline & rs93276I & Chr9:I2665380 & & & & & \\
\hline
\end{tabular}

a Coordinates from HapMap Data Rel. 2 Ia/Phasell, Jan07 on NCBI B35 assembly, dbSNP bI 25.

b Between brackets, ancestral/derived coding of haplotypes; 0 : ancestral, I: derived

c CEU: HapMap North European Caucasians; ASI: HapMap Chinese+Japanese; YRI: HapMap Yorubas.

d Population in which the core haplotype shows longer than expected EHH at a significance value of 0.05 (see Methods).

As regards the nature of selection, the resequencing analysis did not detect any polymorphism in the known proximal regulatory-elements that could be associated with pigmentary differences. However, judging by the localization of the core SNPs identified by the EHH tests it seems likely that, at least in the case of TYRP1, distal regulatory regions (see [37]) are the targets of selection. Selection at the level of gene expression for TYRP1 would be compatible with the observation that the most darkly pigmented individuals seem to have more TYRP1 protein per melanocyte, which has been claimed to be at least partly responsible for the greater tyrosinase activity and melanogenesis reported for dark skin [44]. In addition, it seems likely that non-directional selection is acting too. For instance, overdominance seems compatible with the diversity patterns observed for DCT in Europeans. Similarly, the high frequency of the ancestral TYR haplotype within the Senegalese suggests that purifying selection at this locus may have been of some importance within Africa, although the large number of TYR mutations associated with oculocutaneous albinism 1 (OCA1) (OMIM\#230100) suggest that TYR may be a gene under purifying selection in all populations. Interestingly, Stokowski et al. [45], by means of a multistage genomewide association study, have recently found that polymorphisms in TYR (along with SLC24A5 and SLC45A2) show significant associations with skin-reflectance measurements in a South Asian population. Whether this is a particular characteristic of this population remains to be explored, but altogether, this suggests that a number of different selective regimes may have interacted in a complex way in the evolution of melanogenesis. As a result, the lighter skin pigmentation phenotype in Europeans and East Asians may have been acquired by alternative mechanisms and thus, as previously suggested [11-13,15], light skin would be the result of convergent evolution. Similarly, the diversity profile of Australian Aborigines suggests that this may also be the case for dark skin.

\section{Methods \\ Cells and cell cultures}

All melanocytes used were obtained from human skin. Melanocyte line M0202 was obtained from a lightly pigmented donor by one of us (NS) in the lab. Melanocytes M1 ("Caucasian", 1.5 years old, male donor) and M4 ("Negroid", 4 years old, male donor) were purchased from Promocell (Heidelberg, Germany). The 19-HEM cell line (lightly pigmented) was purchased from Gentaur (Belgium), and NHEM cells (two lightly pigmented and three darkly pigmented) were purchased from Cascade Biologics (Nottinghamshire, UK).

Cell cultures were maintained in an incubator at $37^{\circ} \mathrm{C}$ and $5 \% \mathrm{CO}_{2}$. Melanocyte culture conditions were as indicated by the suppliers. Briefly: a) M0202 cells were initially grown in FETI medium: H-10, 2\% FCS, 1\% Ultroser $\mathrm{G}$ (BioSepra, Ciphergen, France), $4 \mathrm{ng} / \mathrm{ml}$ bFGF, $2 \mathrm{ng} / \mathrm{ml}$ endothelin-1, $5.3 \mathrm{~nm}$ TPA, $0.05 \mathrm{mM}$ IBMX, but were later grown in $\mathrm{H}-10$ supplemented with HEPES $6 \mathrm{mM}, 5 \%$ FBS and MelanoMax (Gentaur), which presumably contains TPA, CT and contains BPE at $40 \mu \mathrm{g} / \mathrm{ml}$ (C. Stefanidis, Gentaur; personal communication); b) M1 and M4 melanocytes were cultured in melanocyte growth medium M2 (Promocell). M2 is a serum-free medium, without PMA (TPA) or other tumor promoting or toxic agents, consisting of a basal medium plus a Supplement Mix. The detailed formulation has not been disclosed by the supplier, but it seems that no BPE is present in this growth medium (Ute Liegibel, PromoCell, personal communication). c) 19-HEM melanocytes were grown in H-10 sup- 
plemented with HEPES $6 \mathrm{mM}, 5 \%$ FBS and MelanoMax (Gentaur); d) NHEM melanocytes culture medium was Cascade Biologics Medium 254 supplemented with 1\% HMGS (containing BPE, FBS, bovine insuline, bovine transferrin, bFGF, hydrocortisone, heparin and PMA) (Cascade Biologics).

The culture medium was changed every two days until the culture was approximately $80-90 \%$ confluent, and everyday thereafter.

M0202, HEM and NHEM melanocytes were passaged (split 1:3) routinely every 10 or 11 days, or harvested when they had reached confluency. The growth rate for M1 and M4 melanocytes was slow, and passaging (1:2) or harvesting at confluency was performed routinely after 2 to 3 weeks. All melanocytes used were from passage 5 to 15 , and all were from normal non-transformed primary cell culture isolates.

\section{Irradiation}

Subconfluence cultures were irradiated once a day for 5 consecutive days with UVA+B $\left(50 \mathrm{~mJ} / \mathrm{cm}^{2}: 25 \mathrm{~mJ} / \mathrm{cm}^{2}\right)$ light in an ICH2 photoreactor (LuzChem, Canada) at $37^{\circ} \mathrm{C}$. These doses assumed an absorbance of the plastic flask of approximately 5\% for UVA and 11\% for UVB (flasks were opaque to UVC), estimated from spectrophotometer absorbance readings at $255 \mathrm{~nm}, 305 \mathrm{~nm}$ and 360 $\mathrm{nm}$ of cuvette-size splinters of the flasks. By trials with murine melanoma cells (B16F10), this dose was shown to have no effect on cell viability. In order to prevent the generation of toxic metabolites, the culture medium was replaced by PBS with magnesium and calcium immediately before irradiation. After irradiation, PBS was replaced again by the culture medium. Irradiation control cultures were subject to the same procedure, except that they were covered by aluminum foil during irradiation. Cultures were harvested $24 \mathrm{~h}$ after the last irradiation dose.

\section{Microarray gene expression}

Immediately after harvesting, cells were resuspended in lysis buffer. Total RNA was obtained following the supplier's protocol (Ambion's total RNA extraction kit), including DNAse treatment. cDNA was synthesized from $2 \mu \mathrm{gr}$ of total RNA using the Affymetrix One-Cycle cDNA Synthesis Kit and following the Affymetrix Expression Analysis Technical Manual. From this cDNA, cRNA was synthesized using the Affymetrix IVT Labeling Kit, which was then purified with the Affymetrix GeneChip Sample Cleanup Module. The purified cRNA (15 $\mu \mathrm{gr})$ was fragmented and hybridized to Affymetrix U133A 2.0 arrays using standard Affymetrix protocols. A total of 18 microarrays from 9 irradiated cell lines (5 from lightly pigmented donors and 4 from darkly pigmented donors) plus their corresponding unirradiated control cultures were analyzed.

\section{Microarray Data Analysis}

Raw data were $\log _{2}$ transformed and quantile normalized using DNAMR v1.1 [46] for R (2.4.1). The Pattern Discovery option of SAM software v3.0 [47] was used to analyze the normalized data. Gene Ontology analysis was done using FatiGO [48].

\section{Quantitative PCR}

To demonstrate that the media composition, in particular the presence/absence of $\mathrm{BPE}$, affects the expression levels of TYR, TYRP1 and DCT we purchased a new melanocyte cell line from Cascade Biologics (lightly pigmented) and grew this cell line in Cascade growth medium 254 supplemented with 1\% HGMS (Cascade + for short). We subcultured and propagated the cells for approximately 3 weeks (three passages). Three days after the third passage we generated 4 subcultures of the same cell line. These 4 subcultures were from now on grown in 6 different media: Medium 1: Cascade +; Medium 2: Cascade medium, PromoCell Supplement (no BPE); Medium 3: PromoCell growth medium plus PromoCell Supplement. Medium 4: PromoCell growth medium plus PromoCell Supplement, plus $0.2 \%(\mathrm{v} / \mathrm{v})$ of a $13 \mathrm{mg} / \mathrm{ml}$ solution of BPE. Cells were grown in these media for four days to allow some adaptation to the new media. Media were refreshed every two days.

After this time, we extracted total RNA from each subculture (Ambion). cDNA was synthesized using the Invitrogen SuperScript First-Strand synthesis system for RT-PCR kit, and then, we quantified the expression of TYR, TYRP1 and DCT for each of these subcultures using the BIO-RAD iQ SYBR green Supermix system in combination with a BIO-RAD iCycler machine. For mRNA quantification the following primers were used: TYR: 5'-AGAATGCTCTGGCTGTTTTG-3' and 5'-TCCATCAGGTTCTTAGAGGAGACAC-3'. For TYRP1: 5'CATGCAGGAAATGTTGCAAGAG-3' and 5'-AGTTTGGGCTTATTAGAGTGGAATC-3'; For DCT: 5'-TATTAGGACCAGGACGCCCC-3' and 5'TGGTACCGGTGCCAGGTAAC-3'. For normalization GADPH was used; primer: 5'-CCTGTTCGACAGTCAGCC3' and 5'-CGACCAAATCCGTTGACTCC-3'. In all cases, annealing temperatures were fixed at $56^{\circ} \mathrm{C}$. For $D C T$, $\mathrm{Mg}^{2+}$ concentration was increased in $1 \mathrm{mM}$ above the standard reaction conditions.

\section{DNA samples and Resequencing}

We have resequenced the following in 116 human chromosomes: a) $4.1 \mathrm{~kb}$ of 5' DCT, including $187 \mathrm{bp}$ of the first intron, the first CDS plus the 5'-UTR and 3,204 bp of the upstream flanking sequence; b) approximately $4 \mathrm{~kb}$ of 
5' TYR, including $17 \mathrm{bp}$ of the first intron, the first CDS plus the 5'-UTR and 2,773 bp of the upstream flanking sequence; and c) approximately $5 \mathrm{~kb}$ of 5' TYRP1, including $433 \mathrm{bp}$ of the first intron, $47 \mathrm{bp}$ of the second intron, the first CDS plus the 5'-UTR and 4,200 bp of the upstream flanking sequence. Sample individuals come from diverse geographical origins and include: 20 chromosomes from Biaka and Mbuti Pygmies (DNA purchased from the European Collection of Cell Cultures, ECACC), 20 chromosomes from Senegalese individuals resident in Spain, 42 European (N. Spain) chromosomes (including 20 chromosomes from melanoma patients), 20 Asian chromosomes including Chinese samples from Coriell Cell Repositories and Chinese residents in Spain, and 14 chromosomes from Australian Aborigines (DNA purchased from the ECACC).

DNA was PCR amplified in overlapping 1 kb long segments and these were resequenced using BigDye 3.1 chemistry and ABI PRISM 3730 and ABI 310 DNA analyzers. Sequences were edited with Genalys v3.3.45a (M. Takahashi). Primers and PCR conditions are available on request. Sequences have been deposited in GenBank, accession numbers DQ821585-DQ821701 for DCT, EF675246-EF675361 for TYR and EF675362-EF675477 for TYRP1.

\section{Estimation of haplotypes}

To solve the haplotypes phase, we first run PHASE [49]. For those pairs of SNPs that did not reach a PHASE probability greater than 0.95 , we solved their phase experimentally by ARMS-PCR and/or cloning (using the TOPO-TA kit from Invitrogene) plus resequencing.

\section{Population parameters and neutrality tests}

After removing polymorphic positions in humans, divergence $(\mathrm{K})$ between a chimp sequence and a human sequence was estimated using K-estimator 6.0 [50]. Initially, population diversity parameters and neutrality statistics like Tajima's D [29] were obtained by means of DnaSP 4.1 [51]. These tests were corrected for demography as specified below. HEW and DHEW tests [32] were carried out using software kindly provided by Kai Zeng.

\section{Optimization of demographic parameters}

To correct for demography in the coalescent neutral simulations of the neutrality tests (excluding HEW and DHEW), we optimized the fit between pairwise $\mathrm{F}_{\mathrm{ST}}$ distributions obtained from real genomic data from the three major geographical human groups in the HapMap project, and those $\mathrm{F}_{\mathrm{ST}}$ distributions from coalescent simulations obtained varying the demographic parameters. The optimization criterion was the p-value of the Kolmogorov-Smirnov D statistic between the real and simulated $\mathrm{F}_{\mathrm{ST}}$ distributions. For the real neutral distribution of the $\mathrm{F}_{\mathrm{ST}}$ statistic [52], we used that obtained previously in [15] in which we selected 43 regions distributed across the autosomal genome that belong to broader regions of low gene density, and which are at least $150 \mathrm{~kb}$ away from the closest exon. Each of these regions spanned an average of $1.96 \mathrm{Mb}$, and in total they account for $84.3 \mathrm{Mb}$. For each region we downloaded the SNP frequency information available from the HapMap browser (data Rel \#20/phase II on NCBI B35 assembly, dbSNP b125) for the 3 major populations (Caucasians: 153,339 SNPS, Yorubas: 123,798 SNPs and Chinese: 33,190 SNPs). We further filtered the number of SNPs to include only those SNPs that: a) were at least $100 \mathrm{~kb}$ away from each other, b) have been genotyped in all three populations and c) at least one of the three major populations had a minor allele frequency (MAF) higher than 0.1. A final list of 546 SNPs satisfied these criteria. We used the ms program [53] for the simulations with 3 populations, with sample sizes equal to those in the HapMap population (120 chromosomes for the African population, 120 for Caucasians and 90 for Asians). As starting points in our simulations to optimize demographic parameters, we used those values described in [54]. Simulations were fixed on one segregating site. These SNPs were matched for a MAF of 0.1 in at least one population.

The mean and 95\% upper limits (between brackets) of the observed $\mathrm{F}_{\mathrm{ST}}$ distributions were [15]: Caucasians-Chinese: 0.08 (0.33); Caucasians-Yorubas: 0.14 (0.47) and Chinese-Yorubas: $0.16(0.45)$. Final optimized values were obtained in the simulations under the following conditions: we used an ancestral population size of 24,000 for the African population (population 1) and 7,700 for both Asians and Caucasians (populations 2 and 3, respectively), with a migration rate matrix $\mathrm{M}_{\mathrm{ij}}=\{0,0.05,0.4$, $0.1,03,0.8,2.5,0\}$ for $i$ and $j$ values from 1 to 3 . Looking back in time, we assumed two bottlenecks with instant population reduction, each followed by a population fusion: one approximately 40,000 years ago, in which the Chinese population reduced its size to approximately one sixth. About 2,000 years after this episode, the Chinese population fuses with the European population. Assuming a generation time of 20 years, this represents an $\mathrm{F}$ value of 0.04 for this bottleneck. A second population bottleneck takes place about 90,000 years ago. On this occasion, the Eurasian population suffers a reduction in size to one sixth of its previous size. About 10,000 years after this bottleneck $(F=0.21)$, the Eurasian population fuses with the African population. The mean and 95\% upper limits (between brackets) of the simulated $\mathrm{F}_{\mathrm{ST}}$ distributions were: Caucasians-Chinese: 0.08 (0.31); Caucasians-Yorubas: $0.15(0.44)$ and Chinese-Yorubas: 0.15 (0.45). The optimized, simulated $\mathrm{F}_{\mathrm{ST}}$ distribution and the real distribution recorded Kolmogorov-Smirnov D values of 0.0356 for Caucasians vs. Asians ( $p$-value 0.891), 
0.0748 for Caucasians vs. Africans ( $p$-value 0.104), and 0.0441 for Asians vs. Africans ( $p$-value 0.683). As both simulated and real data are not statistically different, we used those demographical parameters used in the simulations for the subsequent neutrality tests.

\section{Correction for demography in the neutrality tests}

These demographic and genetic parameters were subsequently used in further simulations for estimating the critical points in Tajima's D. These simulations also allowed us to obtain the distribution of Tajima's D under the genetic and demographic parameters described above.

\section{Extended Haplotype Homozygosity (EHH) test}

For the EHH test, we initially used Sweep 1.0 [55] to scan and select the core haplotypes. We initially focused on those haplotypes that showed both substantial frequency and high EHH values from the core SNPs, in both 5 ' and 3 ' directions. By means of a Perl script, we then calculated the EHH values as in [26] for a region extending about 50 $\mathrm{kb}$ from the closer core SNP. To test the significance of the test, we ran coalescent simulations using the population and demographical parameters described above for the $\mathrm{F}_{\mathrm{ST}}$ distributions, but in this case several aspects are different from our previous approach [15]. First, in this case we included the variable recombination rate information obtained from the HapMap webpage for the particular region tested. In each case, we obtained a discrete number of recombination rate classes using the following approach: for each region we obtained the average and standard deviation (sd) of the distribution of recombination rates obtained from the HapMap link. All recombination values greater than the average plus 2 sd were considered outliers. If these outlier regions were consecutive, a local average was calculated; otherwise, a single outlier value was assigned for that region flanked between the previous and the next recombination values. We then excluded these outliers and repeated the process. In this second round, recombination rates that were higher than the new global average plus 1 sd were considered again as a class each, except if they were consecutive, in which case a local average was estimated. The average of the rest of recombination rates was considered as the background recombination rate, which was used as a reference to estimate the relative intensity of recombination for all other recombination classes.

For the coalescent simulations with heterogeneous recombination rates, we used msHOT [56], a modification of [53] coalescent-based program ( $\mathrm{ms}$ ) for simulating genetic variation data for a sample of chromosomes from a population. Second, in order to obtain a null distribution that reflects a neutral scenario for the chosen core haplotypes, we proceeded as follows: a) in the simulations, the "core" was the set of the first $n$ SNPs, where $n$ is the number of
SNPs in the original core for each case. We then discarded those simulations that did not result in a number of distinct haplotypes (as defined from the simulated core set of SNPs) identical to that observed in the HapMap data using the corresponding observed core SNPs; b) one of the simulated haplotypes had to match in frequency (allowing for a $2 \%$ difference) our observed core haplotype being tested, and in addition, it had to show the same ancestral/derived states for the SNPs composing the core. For the latter, ancestrality was obtained by comparing the corresponding orthologous regions from the chimpanzee genome sequence and the Macaca mulatta genome sequence obtained from the UCSC genome browser [57] or the Ensembl genome browser [58]; c) for each set of simulations that fulfilled these conditions (we typically ran the program until we obtained about 500 simulations satisfying all the conditions), we obtained the 95\% upper percentile of the expected $\mathrm{EHH}$ distribution for each of a series of consecutive $1 \mathrm{~kb}$-long windows spanning the region. We finally declared a core haplotype as under selection if the distance for which the EHH values were equal to 1 for the SNPs in the HapMap data was longer than the distance observed from the $95 \%$ upper percentile distribution of the $\mathrm{EHH}$ values obtained in the simulations.

This approach offers several advantages over other methods currently being used to detect selection. For instance, it allows for a direct comparison with a "neutral" null distribution. In contrast, null distributions obtained from genomic regions are not representative of a homogeneous neutral scenario, but rather the result of heterogeneous evolutionary processes. In addition, it allows statistical inference even when no alternative haplotypes for the same core SNPs are available in order to obtain relative EHH values, as done in other approaches. Finally, ancestral-derived state information and information on the core haplotypes frequency can be incorporated, which helps fine-tune the nature of the elements being compared (observed and simulated data).

\section{Multiple testing corrections}

To control for multiple testing in the $\mathrm{F}_{\mathrm{ST}}$ tests, we used the approach by [59], which sets the false discovery rate (FDR) at a level $\alpha$ by ranking the initial $p$ values in ascending order $P_{(1)} \leq P_{(2)} \leq \ldots \leq P_{(\mathrm{m})}$, with $m$ being the number of tests, and then by specifying $P_{(\mathrm{i})} \leq \alpha \mathrm{i} / m$ as the point below which there is no rejection at an FDR of $\alpha$.

\section{Genealogical relationships among haplotypes}

Graphical representations of the genealogical relationships among haplotypes were estimated by the MedianJoining (MJ) algorithm implemented in Network 4.1.1.2 [60]. When feasible, the ancestral haplotype was inferred 
using parsimony by comparison to the chimp and rhesus macaque sequences.

\section{Test for overdominance}

To evaluate the possibility of overdominance (heterozygote advantage), we scored the ratio of "observed heterozygosity" to "expected heterozygosity" for single SNPs. Observed heterozygosity was estimated by counting heterozygote individuals in the HapMap data set for each SNP in question. The expected heterozygosity was estimated by calculating gene diversity from allele frequencies. Significance for ratio values greater than 1 was obtained by simulation using $m s$ [53].

\section{Authors' contributions}

SA and CR conceived and designed the study. SA analyzed the microarray data, devised the Perl scripts, and conducted most of the population genetic analyses. SA and CR wrote the first draft of the manuscript.

NI did the bulk of the resequencing work and haplotype inference.

DZ contributed significantly to the resequencing work.

ISZ, MDB and NS took responsibility for the cell cultures

JLDP, JLDR and JG participated in the design of the study, obtained the DNA from the melanoma patients and devised the irradiation experiments.

All authors reviewed the manuscript. All authors read and approved the final draft.

\section{Additional material}

\section{Additional file 1}

containing the analyzed Affymetrix U133A v2 gene expression normalized values for the 9 melanocyte cell lines (both control and irradiated with UV Light).

Click here for file

[http://www.biomedcentral.com/content/supplementary/1471-

2148-8-74-S1.7z]

\section{Additional file 2}

containing allele frequencies and genotypes.

Click here for file

[http://www.biomedcentral.com/content/supplementary/1471-

2148-8-74-S2.xls]

\section{Additional file 3}

containing the SNPs and indels submitted to dbSNP.

Click here for file

[http://www.biomedcentral.com/content/supplementary/1471-

2148-8-74-S3.txt]

\section{Acknowledgements}

We thank Celine Derbois (CNG, Evry, France) for her efficient technical assistance. The SGI/IZO-SGlker UPV/EHU (supported by the National Program for the Promotion of Human Resources within the National Plan of Scientific Research, Development and Innovation - European Social Fund, MCyT and Basque Government) is gratefully acknowledged for the generous allocation of computational resources. The authors also wish to thank Garret Hellenthal for kindly providing a modified version of $m s H O T$ and Kai Zeng for the software to implement the HEW and DHEW tests. SA was funded by the Spanish Ministry of Education and Science and by the University of the Basque Country (Ramón y Cajal program). IG is a PhD student supported by the Basque Government. Work funded by grants UE03/A02 and UPV05/I50 from the University of the Basque Country and IP-453-07 from the Basque Government to CR, and Bizkaitek 2003-4 (Provincial Council of Bizkaia) to SA.

\section{References}

I. Bennet D, Lamoreux ML: The color loci of mice - A genetic century. Pigment Cell Res 2003, 16:333-344.

2. Jablonski NG, Chaplin G: The evolution of human skin coloration. J Hum Evol 2000, 39:57-106.

3. Relethford J: Apportionment of Global Human Genetic Diversity Based on Craniometrics and Skin Color. Am J Phys Anthropol 2002, II 8:393-398.

4. Rana BK, Hewett-Emmett D, Jin L, Chang BH, Sambuughin N, Lin M, Watkins S, Bamshad M, Jorde LB, Ramsay M, Jenkins T, Li WH: High polymorphism at the human melanocortin I receptor locus. Genetics 1999, I 5 I: I547-1557.

5. Harding RM, Healy E, Ray AJ, Ellis NS, Flanagan N, Todd C, Dixon C, Sajantila A, Jackson IJ, Birch-Machin MA, Rees JL: Evidence for variable selective pressures at MCIR. Am J Hum Genet 2000, 66:135|-|36|.

6. Mundy $\mathrm{NI}$, Kelly J: Evolution of a pigmentation gene, the melanocortin-I receptor, in primates. Am J Phys Anthropol 2003, | 2 |:67-80.

7. Akey JM, Wang H, Xiong M, Wu H, Liu W, Shriver MD, Jin L: Interaction between the melanocortin-I receptor and $P$ genes contributes to inter-individual variation in skin pigmentation phenotypes in a Tibetan population. Hum Genet 200I, 108:516-520.

8. Soejima M, Tachida H, Ishida T, Sano A, Koda Y: Evidence for recent positive selection at the human AIMI locus in a European population. Mol Biol Evol 2006, 23: 179-188.

9. Kanetsky PA, Swoyer J, Panossian S, Holmes R, Guerry D, Rebbeck TR: A polymorphism in the agouti signaling protein gene is associated with human pigmentation. Am J Hum Genet 2002, 70:770-775.

10. Bonilla C, Boxill LA, Donald SA, Williams T, Sylvester N, Parra EJ, Dios S, Norton HL, Shriver MD, Kittles RA: The 88I8G allele of the agouti signaling protein (ASIP) gene is ancestral and is associated with darker skin colour in African Americans. Hum Genet 2005, I I 6:402-406.

II. Lamason RL, Mohideen MA, Mest JR, Wong AC, Norton HL, Aros MC, Jurynec MJ, Mao X, Humphreville VR, Humbert JE, Sinha S, Moore JL, Jagadeeswaran P, Zhao W, Ning G, Makalowska I, McKeigue PM, O'Donnell D, Kittles R, Parra EJ, Mangini NJ, Grunwald DJ, Shriver MD, Canfield VA, Cheng KC: SLC24A5, a putative cation exchanger, affects pigmentation in zebrafish and humans. Science 2005, 31 0: $1782-1786$.

12. Myles S, Somel M, Tang K, Kelso J, Stoneking M: Identifying genes underlying skin pigmentation differences among human populations. Hum Genet 2007, I 20:613-62I.

13. Norton HL, Kittles RA, Parra E, McKeigue P, Mao X, Cheng K, Canfield VA, Bradley DG, McEvoy B, Shriver MD: Genetic evidence for the convergent evolution of light skin in Europeans and East Asians. Mol Biol Evol 2007, 24:710-722.

14. Voight BF, Kudaravalli S, Wen X, Pritchard JK: A map of recent positive selection in the human genome. PLoS Biol 2006, 4:e72.

15. Izagirre N, García I, Junquera C, de la Rúa C, Alonso S: A scan for signatures of positive selection in candidate loci for skin pigmentation in humans. Mol Biol Evol 2006, 23:1697-1706. 
16. Kunisada H, Nishiwaka S, Nishiwaka SI, Mizoguchi M, Hayashi SI, Tyrrell L, Williams DA, Wang $X$, Longley $B$ J: Murine cutaneous mastocytosis and epidermal melanocytosis induced by keratynocyte expression of transgenic stem cell factor. J Exp Med 1998, I 87:1565-1573.

17. Cooksey CJ, Garratt PJ, Land EJ, Pavel S, Ramsden CA, Riley PA, Smit NP: Evidence of the indirect formation of the catecholic intermediate substrate responsible for the autoactivation kinetics of tyrosinase. J Biol Chem 1997, 272:26226-26235.

18. Ito S: A chemist's view of melanogenesis. Pigment Cell Res 2003, I 6:230-236.

19. García-Borrón JC, Sánchez-Laorden BL, Jiménez-Cervantes C Melanocortin-I receptor structure and functional regulation. Pigment Cell Res 2005, I8:393-410.

20. Buscá R, Ballotti R: Cyclic AMP a key messenger in the regulation of skin pigmentation. Pigment Cell Res 2000, I 3:60-69.

21. Carroll SB, Grenier JK, Weatherbee SD: From DNA to diversity. Molecular genetics and the evolution of animal design London. UK: Blackwell Science; 200I

22. Pastinen $\mathrm{T}$, Hudson T]: Cis-acting regulatory variation in the human genome. Science 2004, 306:647-650.

23. Murisier F, Beerman F: Genetics of pigment cells: lessons from the tyrosinase gene family. Histol- Histopatology 2006 , 2I:567-578

24. Bamshad MJ, Mummidi S, Gonzalez E, Ahuja SS, Dunn DM, Watkins WS, Wooding S, Stone AC, Jorde LB, Weiss RB, Ahuja SK: A strong signature of balancing selection in the $5^{\prime}$ cis-regulatory region of CCR5. Proc Natl Acad Sci USA 2002, 99: I0539-I0544.

25. Rockman MV, Hahn MW, Soranzo N, Zimprich F, Goldstein DB Wary GA: Ancient and recent positive selection transformed opioid cis-regulation in humans. PLoS Biol 2005, 3:e387.

26. D'Orazio JA, Nobuhisa T, Cui R, Arya M, Spry M, Wakamatsu K, Igras V, Kunisada T, Granter SR, Nishimura EK, Ito S, Fisher DE: Topical drug rescue strategy and skin protection based on the role of MCIR in UV-induced tanning. Nature 2006, 443:340-344

27. Abdel-Malek Z, Swope VB, Suzuki I, Akcali C, Harringer MD, Boyce ST, Urabe K, Hearing V]: Mitogenic and melanogenic stimulation of normal human melanocytes by melanotropic peptides. Proc Natl Acad Sci USA 1995, 92: I789-I793.

28. Amae S, Yasumoto KI, Takeda K, Udono T, Takahashi K, Shibahara S: Identification of a composite enhancer of the human tyrosinase-related protein 2/DOPAchrome tautomerase gene. Biochim Biophys Acta 2000, I 492:505-508.

29. Nachman MW, Crowell SL: Estimate of the mutation rate per nucleotide in humans. Genetics 2000, I 56:297-304.

30. Tajima F: Statistical method for testing the neutral mutation hypothesis by DNA polymorphism. Genetics 1989, I 23:585-595.

31. Hudjashov G, Kivisild T, Underhill PA, Endicott P, Sanchez J], Lin AA Shen P, Oefner P, Renfrew C, Villems R, Forster P: Revealing the prehistoric settlement of Australia by $\mathbf{Y}$ chromosome and mtDNA analysis. Proc Natl Acad Sci USA 2007, I 04:8726-8730.

32. Zeng K, Shi S, Wu Cl: Compound tests for the detection of hitchhiking under positive selection. Mol Biol Evol 2007, 24:1898-1908

33. Cavalli-Sforza LL, Menozzi P, Piazza A: The history and geography of human genes Princeton, New Jersey, USA: Princeton University Press; 1994

34. Tajima F: DNA polymorphisms in a subdivided population: the expected number of segregating sites in the two-subpopulation model. Genetics 1989, I 23:229-240.

35. Sabeti PC, Schaffner SF, Fry, Lohmueller J, Varily P, Shamovsky O, Palma A, Mikkelsen TS, Altshuler D, Lander ES: Positive natura selection in the human lineage. Science 2006, 3 I 2:1614-1620.

36. Sabeti PC, Reich DE, Higgins JM, Levine HZ, Richter DJ, Schaffner SF, Gabriel SB, Platko JV, Patterson NJ, McDonald G], Ackerman HC Campbell SJ, Altshuler D, Cooper R, Kwiatkowski D, Ward R, Lander ES: Detecting recent positive selection in the human genome from haplotype structure. Nature 2002, 41 9:832-837.

37. Murisier F, Guichard S, Beerman F: A conserved transcriptional enhancer that specifies Tyrpl expression to melanocytes. Develop Biol 2006, 298:644-655.

38. Kadekaro AL, Kavanagh RJ, Wakamatsu K, Ito S, Pipitione MA, AbdelMalek Z: Cutaneous photobiology. The melanocyte vs. the sun: who will win the final round? Pigment Cell Res 2003, 1 6:434-447.
39. Robins $\mathrm{AH}$ : Biological perspectives on human pigmentation Cambridge Studies in Biological Anthropology. Cambridge, UK: Cambridge University Press; 1991.

40. Jablonski $\mathrm{N}$ : The evolution of human skin and skin colour. Annu Rev Anthropol 2004, 33:585-623.

4I. Montagna W, Prota G, Kenney JA: Black skin: structure and function New York: Academic Press; 1993.

42. Teshima KM, Coop G, Przeworski M: How reliable are empirical genomic scans for selective sweeps? Genome Res 2007 1 6:702-7|2.

43. Tadokoro T, Yamaguchi Y, Batzer J, Coelho SG, Amudzka BZ, Miller SA, Wolber R, Beer JZ, Hearing V]: Mechanisms of skin tanning in different racial/ethnic groups in response to ultraviolet radiation. J Invest Dermatol 2005, I 24: I 326- I332.

44. Alaluf S, Barret K, Blount M, Carter N: Ethnic variation in tyrosinase and TYRPI expression in photoexposed and photoprotected human skin. Pigment Cell Res 2003, 16:35-42.

45. Stokowski RP, Krishna Pant PV, Dadd T, Fereday A, Hinds DA, Jarman C, Filsell W, Ginger RS, Green MR, van der Ouderaa FJ, Cox DR: A Genomewide association study of skin pigmentation in a South Asian population. Am J Hum Genet 2007, 8 I : I I I 9- I I 32.

46. DNAMR [http://www.rci.rutgers.edu/ cabrera/DNAMR/]

47. SAM [http://www-stat.stanford.edu/ tibs/SAM/]

48. Al-Shahrour F, Díaz-Uriarte R, Dopazo J: FatiGO: a web tool for finding significant associations of Gene Ontology terms with groups of genes. Bioinformatics 2004, 20:578-580.

49. Stephens M, Donnelly P: A comparison of Bayesian methods for haplotype reconstruction from population genotype data. Am J Hum Genet 2003, 73: I I62-I I69.

50. Comeron J: K-estimator: Calculation of the number of nucleotide substitutions per site and the confidence intervals. Bioinformatics 1999, I5:763-764.

51. Rozas J, Sanchez-DelBarrio JC, Messeguer X, Rozas R: DnaSP, DNA polymorphism analyses by the coalescent and other methods. Bioinformatics 2003, 19:2496-2497.

52. Weir BS, Cockerham CC: Estimating F-statistics for the analysis of population structure. Evolution 1984, 38: I358-1370.

53. Hudson RR: Generating samples under a Wright-Fisher neutral model of genetic variation. Bioinformatics 2002, I 8:337-338.

54. Schaffner SF, Foo C, Gabriel S, Reich D, Daly D, Altshuler D: Calibrating a coalescent simulation of human genome sequence variation. Genome Res 2006, I 5:1576-1583.

55. SWEEP [http://www.broad.mit.edu/mpg/sweep/index.html]

56. Hellenthal G, Stephens M: msHOT: modifying Hudson's ms simulator to incorporate crossover and gene conversion hotspots. Bioinformatics 2007, 23:520-52I.

57. UCSC genome browser [http://genome.ucsc.edu/cgi-bin/hgGate way]

58. Ensembl genome browser [http://www.ensembl.org/index.html]

59. Benjamini $Y$, Hochberg $Y$ : Controlling the false discovery rate a practical and powerful approach to multiple testing. J Roy Stat Soc 1995, B57:289-300.

60. Bandelt $\mathrm{H}$, Forster $\mathrm{P}, \mathrm{Röhl} \mathrm{A}$ : Median-joining networks for inferring intraspecific phylogenies. Mol Biol Evol 1999, 16:37-48.
Publish with Bio Med Central and every scientist can read your work free of charge

"BioMed Central will be the most significant development for disseminating the results of biomedical research in our lifetime. "

Sir Paul Nurse, Cancer Research UK

Your research papers will be:

- available free of charge to the entire biomedical community

- peer reviewed and published immediately upon acceptance

- cited in PubMed and archived on PubMed Centra

- yours - you keep the copyright
BiolMedcentral 